\title{
A COMPLETE CENSUS OF $4 \times 4$ MAGIC SQUARES*
}

\author{
BY D. N. LEHMER
}

A complete enumeration of magic squares of order 3 is easy to make and the result turns out to be 72 . In this enumeration two squares are considered different if they do not have the same numbers in corresponding cells. In this and in the study of $4 \times 4$ squares we define a magic square as one in which the elements in every row and column add up to the same sum, this sum being given for the square of order $n$ by the formula

$$
\frac{n\left(n^{2}+1\right)}{2} \text {. }
$$

With this definition a magic square remains magic after any permutation of the rows among themselves, or of the columns among themselves, or by an interchange of rows and columns. This associates with any magic square a set of $2(n !)^{2}$ squares all of which are magic.

By a permutation of the rows and columns of any square the largest entry in the square may be transferred to any particular cell; in particular it may be placed in the lower left hand corner. The other rows and columns may then be permuted so that the entries in the bottom line and in the left hand column read in descending order of magnitude. Also by an interchange of rows and columns, if necessary, the element in the bottom row next to the left hand corner may be made larger than the element in the left hand column which is next above the left hand corner. No further adjustments are then possible and the square will be said to be normalized. Thus the square

\begin{tabular}{|c|c|c|c|c|c|c|c|c|}
\hline 22 & 8 & 11 & 19 & & 3 & 21 & 19 & 12 \\
\hline 7 & 15 & 18 & 21 & & 6 & 4 & 22 & 20 \\
\hline $6 \quad 14$ & 17 & 25 & 3 & becomes & 14 & 7 & 5 & 23 \\
\hline 16 & 24 & 2 & 10 & & 17 & 15 & 8 & 1 \\
\hline 23 & 1 & 9 & 12 & & 25 & 18 & 11 & 9 \\
\hline
\end{tabular}

after normalization;

* Presented to the Society, November 29, 1929, and June 23, 1933. 
It is clear that two normalized squares which do not agree entry for entry can not be transformed one into the other by any permutations of rows or of columns, or by interchange of rows and columns. To find the total number of squares of order $n$ all we need to find is the number of normalized squares. This number, multiplied by $2(n !)^{2}$ will give the total number of squares for that order.

To exhibit the method used in the discussion of $4 \times 4$ squares we derive the normalized squares of order three. The sum in each row and column must be 15 . We assume the entry 9 in the lower left hand corner. The other two entries in the bottom row and in the left hand column must add to $15-9=6$. The only partitions of 6 obtainable from the numbers $1,2,3,4,5,6,7,8$, the numbers in the partition being distinct, are $5+1$ and $4+2$. For the normalized square, therefore, we must write the bottom row $9,5,1$ and the left hand column 9, 4, 2. This leaves only the numbers $3,6,7,8$ with which to complete the square. The two remaining numbers in the second column must add to 10 , and the two in the second row to 11 . The only partition of 10 available is $7+3$ and the only partition 11 available is $8+3$. This indicates that the cell in which these two lines intersect must be filled with the number 3 . We get thus the single normalized square of order 3 to be

$\begin{array}{lll}2 & 7 & 6 \\ 4 & 3 & 8 \\ 9 & 5 & 1\end{array}$

All other magic squares of order 3 are therefore obtainable from this single one by permutation of its rows and columns and by interchanging its rows and columns. The number of such transformations being $2(3 !)^{2}=72$, the total number of magic squares of order 3 is therefore 72 .

For the $4 \times 4$ squares the magic sum is 34 . After placing 16 in the lower left hand corner the sum of the three remaining numbers in the first row and in the first column must be $34-16=18$. There are 19 partitions of 18 using the numbers from 1 to 15 , if the numbers in the partitions are assumed distinct as they are in this case. They are $(15,2,1),(14,3,1),(13,4,1),(12,5,1)$, $(11,6,1),(10,7,1),(9,8,1),(13,3,2),(12,4,2),(11,5,2)$, $(10,6,2),(9,7,2),(11,4,3),(10,5,3),(9,6,3),(8,7,3)$, 
$(9,5,4),(8,6,4)$ and $(7,6,5)$. Every normalized magic square must then have one of these triads for the numbers (besides the number 16) in its first row and in its first column. Moreover having selected a triad for the bottom row the triad for the first column must be made up of numbers distinct from those in the triad first selected, and the largest number in it must be smaller than the largest number in the chosen triad. Thus starting with the triad $(15,2,1)$ all the above triads which contain 1 or 2 are not available and there are only seven left, namely, $(11,4,3)$, $(10,6,2),(9,6,3),(8,7,3),(8,6,4),(7,6,5)$. Similarly for the other choices of the triad in the bottom row. It is observed that no triad for the column can be used with a triad chosen for the bottom row if it precedes it in the above list. Thus, for example, for the triad $(10,7,1)$, as a choice for the bottom row we can use only three triads $(9,6,3),(9,5,4),(8,6,4)$, and for the triad $(9,5,4)$ there is only one available: $(8,7,3)$.

These combinations of triads in the bottom line with triads in the first column (67 in all) must each be examined for possible magic squares. The method is analogous to the one used in the above discussion for $3 \times 3$ squares and will be clear from the example of the first case in which we have the triad $(15,2,1)$ for the bottom row and $(11,4,3)$ for the first column. These triads use up the numbers $1,2,3,4,11,15$ and 16 . The remaining numbers are $5,6.7,8,9,10,12,13,14$. Out of these a triad must be selected for the second column which shall give a sum $34-15=19$. The only such is $(8,6,5)$. A triad must also be selected for the second row with a sum 23 . The only ones are $(12,6,5),(10,8,5),(10,7,6),(9,8,6)$. But the vertical column and the horizontal row must have one and only one element in common. This rules out of consideration the 1 st, $2 \mathrm{~d}$ and 4 th of the above triads, and we have left only the triads $(8,6,5)$ and $(10,7,6)$ to consider, with the common element 6 which must therefore be located in the intersection cell. The other elements must then be suitably placed and the result is the two squares:

$\begin{array}{rrrrlrrrr}3 & 8 & 9 & 14 & & 3 & 5 & 12 & 14 \\ 4 & 5 & 13 & 12 & & 4 & 8 & 13 & 9 \\ 11 & 6 & 10 & 7 & \text { and } & 11 & 6 & 7 & 10 \\ 16 & 15 & 2 & 1 & & 16 & 15 & 2 & 1\end{array}$


The discussion for the other cases may be made in the same way. The following table gives for each bottom line triad the set of triads available for the first column, the subscript indicating the number of squares obtained from that particular choice of triads.

ottom

line

triads

Available first column triads

$(15,2,1)(11,4,3)_{2},(10,5,3)_{12},(9,6,3)_{11},(9,5,4)_{11},(8,7,3)_{2}$, $(8,6,4)_{3},(7,6,5)_{4}$.

$(14,3,1)(12,4,2)_{3},(11,5,2)_{13},(10,6,2)_{7},(9,7,2)_{13},(9,5,4)_{20}$, $(8,6,4)_{2},(7,6,5)_{1}$.

$(13,4,1)(11,5,2)_{14},(10,6,2)_{2},(10,5,3)_{10},(9,7,2)_{11},(9,6,3)_{14}$, $(8,7,3)_{5},(7,6,5)_{2}$.

$(13,3,2)(12,5,1)_{14},(11,6,1)_{15},(10,7,1)_{11},(9,8,1)_{22},(9,5,4)_{7}$, $(8,6,4)_{5},(7,6,5)_{0}$.

$(12,5,1)(11,4,3)_{8},(10,6,2)_{3},(9,7,2)_{20},(9,6,3)_{13},(8,7,3)_{3}$, $(8,6,4)_{2}$.

$(12,4,2)(11,6,1)_{4},(10,7,1)_{6},(10,5,3)_{4},(9,8,1)_{2},(9,6,3)_{1}$, $(8,7,3)_{0},(7,6,5)_{0}$.

$(11,6,1)(10,5,3)_{14},(9,7,2)_{15},(9,5,4)_{14},(8,7,3)_{5}$.

$(11,5,2)(10,7,1)_{14},(9,8,1)_{14},(9,6,3)_{5},(8,7,3)_{0},(8,6,4)_{5}$.

$(11,4,3)(10,7,1)_{3},(10,6,2)_{1},(9,8,1)_{4},(9,7,2)_{0},(7,6,5)_{0}$.

$(10,7,1)(9,6,3)_{14},(9,5,4)_{11},(8,6,4)_{1}$.

$(10,6,2)(9,8,1)_{2},(9,5,4)_{2},(8,7,3)_{1}$.

$(10,5,3)(9,8,1)_{13},(9,7,2)_{7},(8,6,4)_{0}$.

$(9,8,1) \quad(7,6,5)_{8}$.

$(9,7,2) \quad(8,6,4)_{7}$.

$(9,5,4) \quad(8,7,3)_{6}$.

It is seen that there are in all 468 normalized squares of order 4 , and multiplying this number by $2(4 !)^{2}=1152$, we get for the total number of magic squares of order 4 the number 539,136 .

The University of California 